\title{
Effects of Powder Shape and Processing Parameters on Heat Dissipation of Heat Pipes with Sintered Porous Wicks
}

\author{
Yech-Ju Lin*1 and Kuen-Shyang Hwang*2 \\ Department of Materials Science and Engineering, National Taiwan University, \\ 1, Roosevelt Rd., Sec. 4, Taipei 106, Taiwan, R. O. China
}

The thermal performance of sintered-type heat pipes can be determined from their permeability, capillary pressure, and capillary speed. These characteristics are closely related to the pore structure, which is influenced by the powder used. To investigate the effects of powder shape on the heat dissipation of a heat pipe, gas atomized, water atomized, and electrolytic copper powders were used in this study. The results showed that the gas atomized spherical powder, despite having the lowest porosity, provided the highest permeability and capillary speed and thus the best heat dissipation. The water atomized irregular powder had a smaller permeability, slightly higher capillary speed, and better thermal performance compared to dendritic electrolytic powder. These results suggest that capillary speed is favorable over the permeability for evaluating whether a copper powder is suitable for heat pipe applications or not. The geometrical factor in the Kozeny-Carman permeability equation, which takes into account the effective pore length, pore surface roughness, and tortuosity, could vary from the 250 of the spherical powder to the 3108 of the dendritic powder for compacts with similar permeabilities, showing the effect of powder shape. The processing parameters, compacting pressure and sintering temperature, were also important. Compacts that were loose-powder-sintered at high temperatures showed higher permeability than those using compaction and low temperature sintering due to the differences in the pore surface roughness. These results demonstrate that the thermal performance of heat pipes is closely related to the powder shape and the process used, in addition to the effects of particle size and particle size distribution. [doi:10.2320/matertrans.M2009152]

(Received April 24, 2009; Accepted July 6, 2009; Published August 19, 2009)

Keywords: copper powder, powder shape, capillary speed, permeability, heat dissipation, sintering, heat pipe

\section{Introduction}

Heat pipes with sintered porous structures have been used widely to resolve the thermal management problems of electronic devices, such as notebook computers. ${ }^{1)}$ To provide effective cooling, the water at one end of the sealed heat pipe, which is under partial vacuum, evaporates into vapor that cools the hot device by using the latent heat, and the water vapor then passes through the hollow channel in the core of the heat pipe to the cold end, where the water vapor condenses. This condensed water is then transported back to the hot evaporator section through the capillary pressure in porous copper wicks, which are sinter-bonded to the outer copper tubing. ${ }^{1)}$ To ensure high heat dissipation, high thermally conductive copper tubing, high enough vacuum in the heat pipe, rapid cooling at the condenser end, and fast enough supply of water through the copper wicks are all required. This study presents the results of how powder shape affects the water supply rate in the heat pipe.

To provide effective water supply, the porous structure must contain large amounts of pores so that enough water can be supplied continuously from the condenser section to the evaporator section. In addition, the pore size must be small enough so that the capillary pressure is high enough to draw the water through the porous wick, sometimes even against gravity. These requirements can be assessed by the permeability, capillary pressure, and capillary speed, which can be described by the following equations. ${ }^{1-6)}$

$$
K=\frac{D^{2} \varepsilon^{3}}{C(1-\varepsilon)^{2}}
$$

$$
\begin{aligned}
P & =\gamma_{\mathrm{lv}} \cos (\theta) S \rho\left(\frac{1-\varepsilon}{\varepsilon}\right) \\
\frac{d h}{d t} & =\left(\frac{r^{2}}{8 \tau \eta h}\right)\left[\frac{\gamma_{\mathrm{lv}} \cos \theta S \rho(1-\varepsilon)}{\varepsilon}-\rho g h\right]
\end{aligned}
$$

In eq. (1), which is commonly referred to as the KozneyCarman equation, the $D$ is the particle size, $\varepsilon$ the porosity, and $C$ a geometrical factor depending on the powder and process used and the resulting tortuosity of the pore structure. In the capillary pressure $(P)$ equation, $\gamma_{\mathrm{lv}}$ is the surface tension at the liquid-vapor interface, $\theta$ the contact angle, $S$ the specific surface area, and $\rho$ the density of the solid. In eq. (3), $h$ is the capillary rise distance, $r$ the effective pore radius, $g$ the gravitational acceleration, $\tau$ the tortuosity, and $\eta$ the dynamic viscosity of water.

Of these three equations, the capillary speed has been recommended for evaluating whether a copper powder is suitable for heat pipe applications or not. ${ }^{6)}$ To use the capillary speed, eq. (3), the effective pore radius $r$ is a critical factor, and it can be measured from the capillary rise height, ${ }^{2,3,7)}$ mercury porosimetry data, ${ }^{7,8}$ or quantitative metallography result; ${ }^{9)}$ or, alternatively, estimated from the following equation for the so-called hydraulic radius: ${ }^{10)}$

$$
\begin{aligned}
r_{\mathrm{h}} & =\frac{\text { pore volume }}{\text { pore surface area }} \\
& =\frac{V_{\mathrm{p}}}{S \rho V_{\mathrm{s}}}
\end{aligned}
$$

where $V_{\mathrm{p}}$ and $V_{\mathrm{s}}$ are the volumes of pores and solids, respectively. Thus,

$$
r_{\mathrm{h}}=\frac{\varepsilon}{S \rho(1-\varepsilon)}
$$

Since the specific surface area of a powder can be approximated by

\footnotetext{
${ }^{* 1}$ Graduate Student, National Taiwan University

${ }^{* 2}$ Corresponding author, E-mail: kshwang@ntu.edu.tw
} 


$$
S=\frac{6 \lambda}{\rho D}
$$

where $\lambda$ is a particle shape factor $(\lambda=1$ for spherical powder), eq. (6) becomes:

$$
r_{\mathrm{h}}=\frac{\varepsilon D}{6 \lambda(1-\varepsilon)}
$$

Thus, eq. (3) can be restated as:

$$
\frac{d h}{d t}=\left(\frac{r_{\mathrm{h}}^{2}}{8 \tau \eta h}\right)\left[\frac{6 \lambda \gamma_{\mathrm{lv}} \cos \theta(1-\varepsilon)}{D \varepsilon}-\rho g h\right]
$$

or

$$
\frac{d h}{d t}=\left[\frac{(\varepsilon D)^{2}}{288 \lambda^{2}(1-\varepsilon)^{2} \tau \eta h}\right]\left[\frac{6 \lambda \gamma_{\mathrm{lv}} \cos \theta(1-\varepsilon)}{D \varepsilon}-\rho g h\right]
$$

Since a large particle size results in a large pore size, eq. (9) and (10) suggest that coarse powders are favorable for the capillary speed and thus the heat dissipation. This postulation was confirmed by Lin and Hwang who demonstrated that coarse spherical powders with a mean particle size, $\mathrm{D}_{50}$, of $128 \mu \mathrm{m}$ has a much faster capillary rise rate than the smaller powders, which have a $\mathrm{D}_{50}$ of 92 and $\left.65 \mu \mathrm{m} .{ }^{6}\right)$ The powder with a narrower particle size distribution was also shown to be more favorable.

In addition to the particle size and particle size distribution, the tortuosity factor, $\tau$, and particle shape factor, $\lambda$, in eq. (10) also indicate that the morphology of the pore, such as the pore shape, surface roughness, and pore length, are critical features too. These features could be influenced by the powder and/or the processing parameters used. For example, two powders with the same particle size but with different shapes could have similar porosity but different pore shapes after sintering. Furthermore, when different temperatures are used for sintering, the sintered densities of two compacts that are made with the same powder could be adjusted to the same value, but the pore shape and surface roughness could be quite different. As a result, different effective pore radii and tortuosities, which have been estimated to be in the range of $\sqrt{2}$ to $7,5,11-13)$ will be attained. Leong et al. demonstrated by using high temperature sintering that a more regular pore shape and less small pores were obtained, characteristics which are favorable for more effective fluid flow. But the high sintered density resulting from the high temperature sintering could equalize the favored effect of the large pore size and regular pore shape and give a low permeability. ${ }^{4)}$ The capillary performance of flat heat pipes with microgrooves was also shown to be more effective when the wetting distance was increased with chemical etching to form microcavities on the surface. ${ }^{14)}$ These previous studies have suggested that the powder shape and processing parameters will influence the pore characteristics, but their effects on the overall heat pipe performances were still not clear. Since the effects of surface roughness, porosity, and pore size, which are determined by the powder shape and processing parameters selected, are still not well understood, a systematic study on these subjects is desirable. In this work, three $\mathrm{Cu}$ powders with different powder shapes and two processing parameters, compacting pressure and (a)

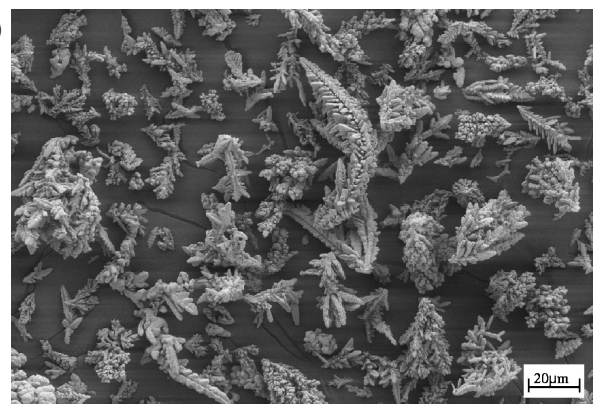

(b)

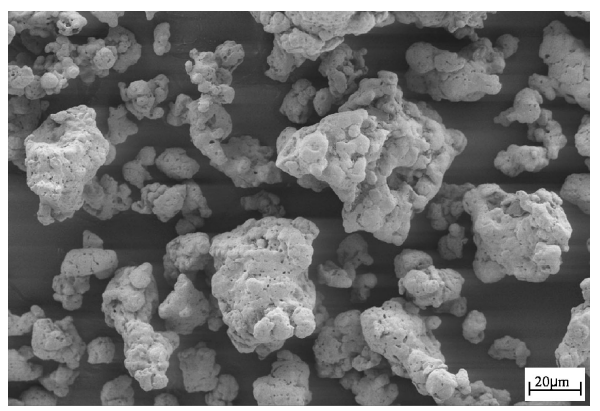

(c)

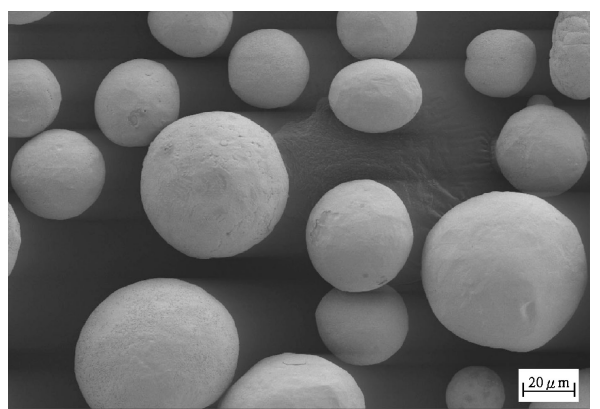

Fig. 1 The morphologies of the copper powders used in this study. (a) Electrolytic powder with dendritic shape, (b) water atomized powder with irregular shape, and (c) gas atomized powder with spherical shape.

sintering temperature, were used, and their effects on the heat dissipation of heat pipes were examined.

\section{Experimental Procedure}

Gas atomized, water atomized, and electrolytic copper powders, which were spherical, irregular, and dendritic in shape, respectively, were selected in this study. The mean particle sizes, $\mathrm{D}_{50}$, of the selected dendritic, irregular, and spherical powders were close, at $21.4,46.2$, and $65.4 \mu \mathrm{m}$, respectively. The morphologies and characteristics of these powders are shown in Fig. 1 and Table 1, respectively. Since the loose powder sintering process was used in this study, the green specimens were prepared by tapping powders in a mold or a container. This green density is very close to the tap density of the powder that has been vibrated for a prolonged period. Thus, the tap densities, which were measured following the ASTM B-527 standard, are also included in Table 1.

For the permeability test, cylindrical specimens were prepared by filling $\mathrm{Cu}$ powders in a graphite mold cavity, $13 \mathrm{~mm}$ in diameter and $14 \mathrm{~mm}$ deep. When different sintered densities were needed, the sintering temperature was adjusted from 973 to $1123 \mathrm{~K}$ in hydrogen and then loosely sintered at 
Table 1 The characteristics of the gas atomized, water atomized, and electrolytic copper powders used in this study.

\begin{tabular}{lccc}
\hline & Electrolytic & $\begin{array}{c}\text { Water } \\
\text { Atomized }\end{array}$ & $\begin{array}{c}\text { Gas } \\
\text { Atomized }\end{array}$ \\
\hline Designation & CH-M13 & Cu-200 & $153 \mathrm{~A}$ \\
Shape & Dendritic & Irregular & Spherical \\
Mean Particle Size, $\mu \mathrm{m}$ & 21.4 & 46.2 & 65.4 \\
(Laser Light Scattering) & & & \\
Apparent Density, $\mathrm{kg} / \mathrm{m}^{3}$ & $1.54 \times 10^{3}$ & $3.05 \times 10^{3}$ & $5.21 \times 10^{3}$ \\
Tap Density, $\mathrm{kg} / \mathrm{m}^{3}$ & $2.17 \times 10^{3}$ & $3.52 \times 10^{3}$ & $5.62 \times 10^{3}$ \\
Flow Rate, $\mathrm{s} / 0.05 \mathrm{~kg}$ & - & 33.67 & 10.83 \\
Oxygen, mass\% & 0.274 & 0.276 & 0.106 \\
Supplier & ECKART & Chi-Lien & ACuPowder \\
\hline
\end{tabular}

that temperature for $3.6 \mathrm{ks}$. For the heat dissipation test, flat specimens were made by pouring copper powders into a stamped rectangular container, which was made of $0.2 \mathrm{~mm}$ thick oxygen-free $\mathrm{Cu}$ sheets. The cavity was $200 \mathrm{~mm}$ long, $19.5 \mathrm{~mm}$ wide, and $1.4 \mathrm{~mm}$ deep. The $\mathrm{Cu}$-powder-filled container was heated at $0.083 \mathrm{~K} / \mathrm{s}$ to $1123 \mathrm{~K}$ in hydrogen and sintered for $3.6 \mathrm{ks}$. After sintering, the sintered density was calculated based on the weight and volume of the specimen.

To investigate the effect of surface roughness of pores on permeability, two groups of compacts with the same sintered density of $4.80 \times 10^{3} \mathrm{~kg} / \mathrm{m}^{3}$ were produced using the same water atomized $\mathrm{Cu}$ powders. The first group was pressed using a pressure of $27.5 \mathrm{MPa}$ and then sintered at a low temperature of $973 \mathrm{~K}$ for $3.6 \mathrm{ks}$. These low-temperaturesintered specimens were designated as LT specimens. The second group was produced using the loose powder sintering technique, without any compaction, and the sintering was carried out at $1073 \mathrm{~K}$ for $3.6 \mathrm{ks}$. These loose-powder-sintered specimens were designated as high temperature (HT) specimens. To examine these LT and HT specimens under a scanning electron microscope (JSM-T100, JEOL Co., Tokyo, Japan), the sintered parts were immersed in liquid nitrogen for $0.6 \mathrm{ks}$ and then fractured so that the true pore structure could be retained with little deformation.

To understand the effect of powder shape on the heat dissipation performance, the permeability and capillarity of the sintered porous $\mathrm{Cu}$ wicks were examined. The permeability was measured by calculating the amount of water that passed through the cylindrical specimen under a fixed pressure head of $147.5 \mathrm{mmHg}$, which resulted from the height difference between the water bath and the specimen. To compare the capillarity, the lower end of the flat rectangular specimen was immersed perpendicularly into water by about $5 \mathrm{~mm}$ and the height of the water was recorded using the litmus paper. The climbing speed of the water was then calculated from the water height data. This speed, along with the porosity, determines the rate of water supply in the heat pipe and is thus directly related to the amount of heat dissipated. To understand the effect of pore size on permeability and capillary pressure, the pore size distribution in the sintered porous $\mathrm{Cu}$ wick was analyzed using a mercury porosimeter (Auto Pore IV 9500, Micromeritics Co., Norcross, Georgia). In addition to the mercury porosimetry analysis, the maximum pore size was also measured using the bubble test method following the ASTM E-128 standard.

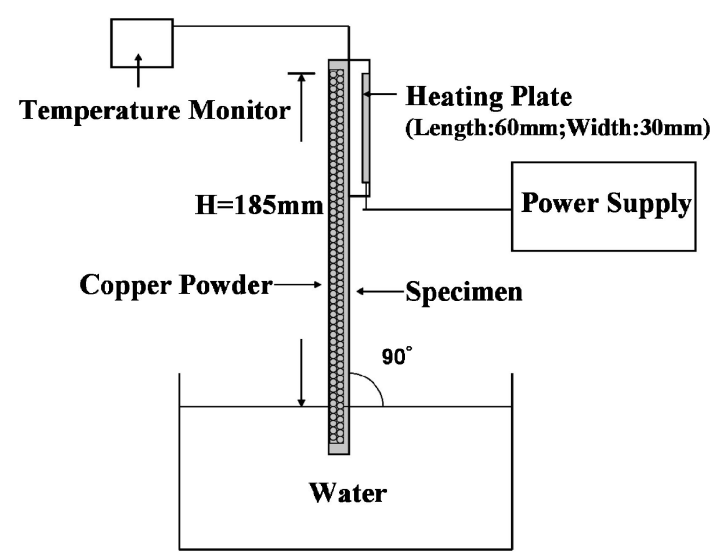

Fig. 2 The set-up of the apparatus for measuring the heat dissipation of the sintered porous plate.

To simulate the heat dissipation of a flat heat pipe, an apparatus as shown in Fig. 2 was prepared. One end of the flat specimen was immersed in water to function as the condenser, and the other end was clamped to a $60 \mathrm{~mm}$ long and $30 \mathrm{~mm}$ wide heating plate. A thin layer of thermal glue was applied to ensure good contact between the heating plate and the specimen, which were held together with a C-clamp. The performance of the heat dissipation was evaluated by comparing the temperature at the evaporator end under a fixed power input and by observing whether a dry-out phenomenon occurred.

\section{Results and Discussion}

\subsection{Effect of powder shape on permeability}

Figure 3 shows the flow rates of water in the $\mathrm{Cu}$ powder compacts that were sintered at $973 \mathrm{~K}$ and $1123 \mathrm{~K}$. The densities of the dendritic, irregular, and spherical powder compacts that were loose-powder-sintered at $1123 \mathrm{~K}$ were $2.77,4.92$, and $5.35 \times 10^{3} \mathrm{~kg} / \mathrm{m}^{3}$, respectively. The corresponding permeabilities were $0.80 \times 10^{-12}, 0.34 \times 10^{-12}$, and $4.50 \times 10^{-12} \mathrm{~m}^{2}$, and the resultant $C$ values in eq. (1) were 1905, 1909, and 176. These curves demonstrate that the spherical powder yielded the highest permeability, even though it had high sintered density, followed by the dendritic powder and the irregular powder.

Also shown in Fig. 3 are the curves with similar permeabilities. When dendritic powder was loose-powdersintered at $973 \mathrm{~K}$, the density was $1.78 \times 10^{3} \mathrm{~kg} / \mathrm{m}^{3}$ and the permeability was $1.92 \times 10^{-12} \mathrm{~m}^{2}$. To attain this permeability, or one close to it, the irregular powder was loosepowder-sintered at $973 \mathrm{~K}$ and the density was about $3.59 \times 10^{3} \mathrm{~kg} / \mathrm{m}^{3}$. For the spherical powder, the density was about $5.61 \times 10^{3} \mathrm{~kg} / \mathrm{m}^{3}$, which was made by tapping the powder-filled graphite mold and then sintering at $1123 \mathrm{~K}$. With these porosity and particle size data, as summarized in Table 2, the geometrical factor, $C$, in the permeability eq. (1) could be calculated and used as an indicator to describe the variations in the pore structure. As shown in Table 2, the $C$ value of the spherical gas atomized powder is 250 , close to the theoretical value of 180 for spherical particles. ${ }^{3,4,11)}$ However, the $C$ values of the water atomized and electrolytic powders were very high, at 1445 and 3108, respectively. 

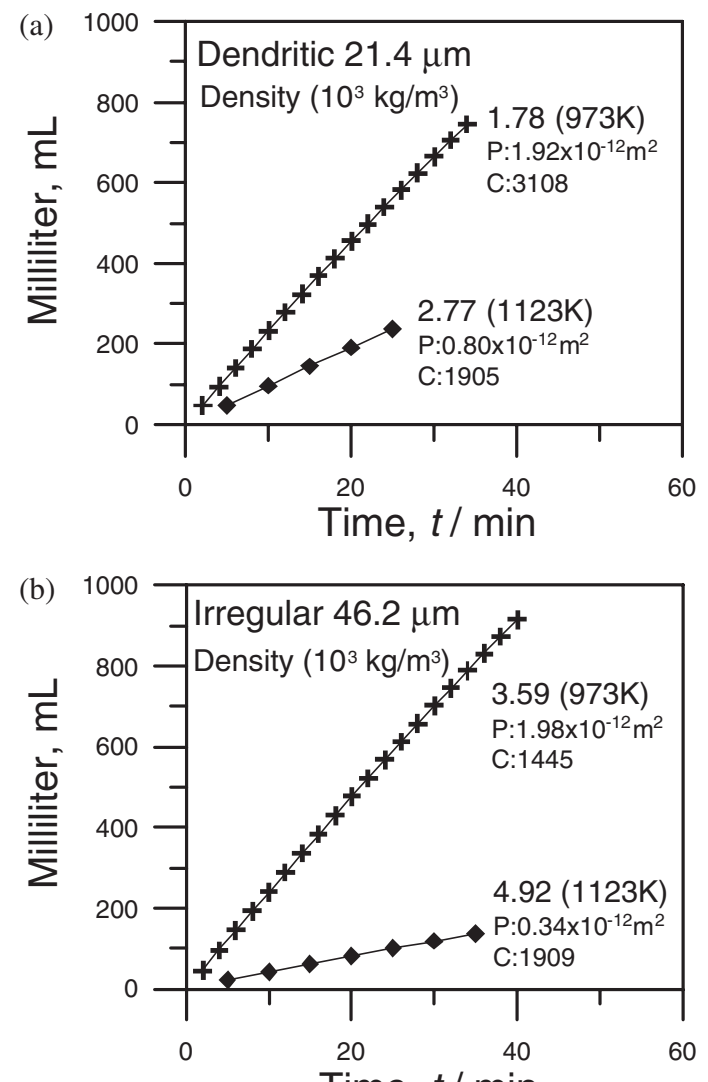

Time, $t / \min$

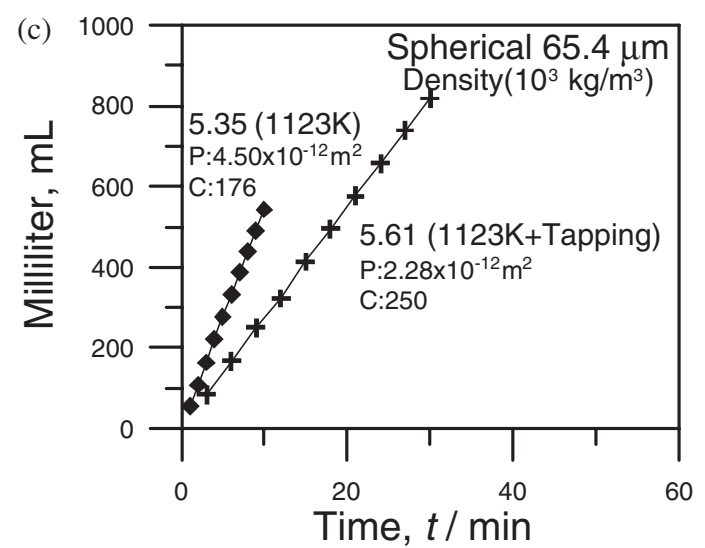

Fig. 3 The flow rate of the compact increases as the sintered density decreases. (a) Electrolytic powder with dendritic shape, (b) water atomized powder with irregular shape, and (c) gas atomized powder with spherical shape.

Table 2 Comparison of the particle size, sintered density, and porosity of the sintered cylindrical compacts having similar permeability.

\begin{tabular}{lcccccc}
\hline & $\begin{array}{c}\text { Particle } \\
\text { Size, } \\
\mu \mathrm{m}\end{array}$ & $\begin{array}{c}\text { Sintering } \\
\text { Temp., } \\
\mathrm{K}\end{array}$ & $\begin{array}{c}\text { Sintered } \\
\text { Density, } \\
\mathrm{kg} / \mathrm{m}^{3}\end{array}$ & $\begin{array}{c}\text { Porosity, } \\
\%\end{array}$ & $\begin{array}{c}\text { Permeability, } \\
10^{-12} \mathrm{~m}^{2}\end{array}$ & $\begin{array}{c}C \text { in } \\
\text { eq. (1) }\end{array}$ \\
\hline Dendritic & 21.4 & 973 & $1.78 \times 10^{3}$ & 80.1 & 1.92 & 3108 \\
Irregular & 46.2 & 973 & $3.59 \times 10^{3}$ & 59.9 & 1.98 & 1445 \\
Spherical & 65.4 & 1123 & $5.61 \times 10^{3}$ & 37.4 & 2.28 & 250 \\
\hline
\end{tabular}

To elucidate the causes of this difference in $C$ values, the variations in density and particle size need to be minimized so that the effects of other factors, such as powder shape, can be examined more exclusively. Thus, the three powders were

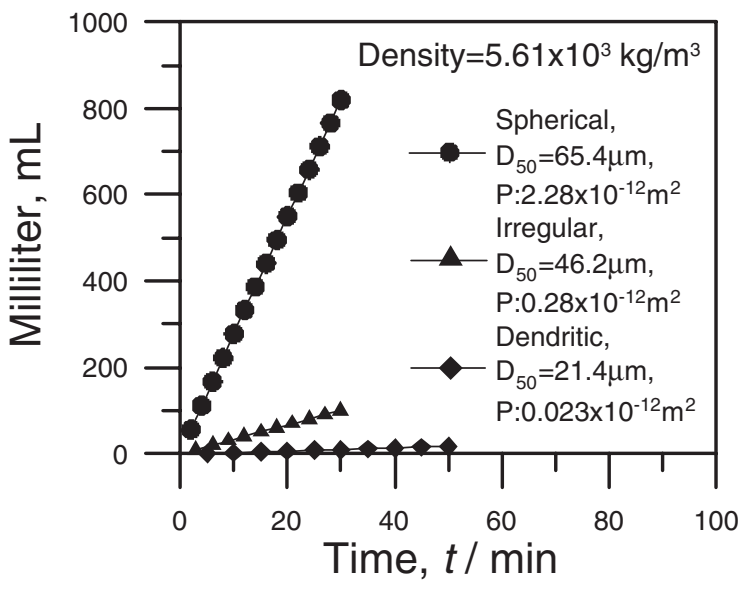

Fig. 4 The flow rate of the specimens with the same density but with different powder shapes.

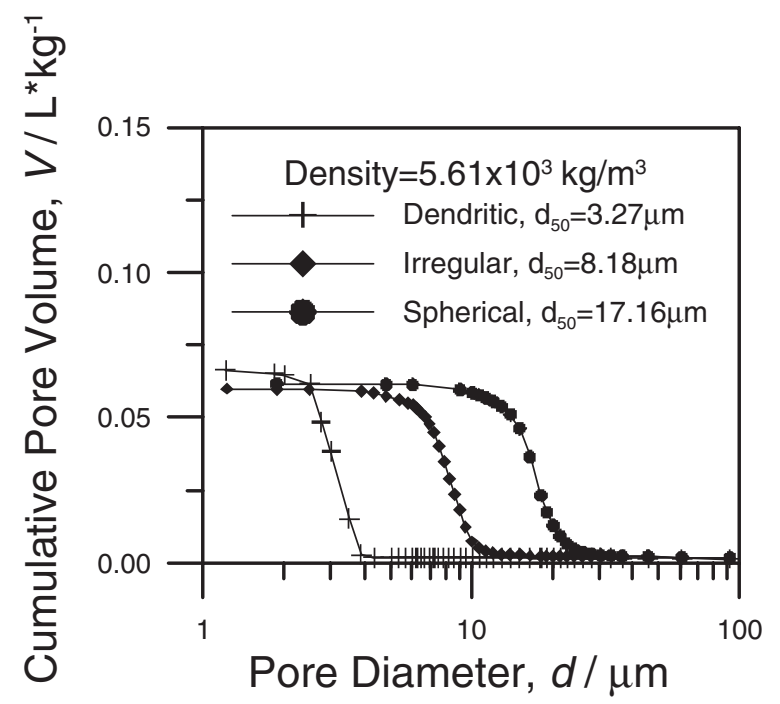

Fig. 5 The pore size distributions of the sintered compacts that were prepared using different kinds of powders.

made into cylindrical compacts with the same density of $5.61 \times 10^{3} \mathrm{~kg} / \mathrm{m}^{3}$. Figure 4 shows that the spherical gas atomized powder produced the highest permeability, $2.28 \times 10^{-12} \mathrm{~m}^{2}$, followed by the $0.28 \times 10^{-12} \mathrm{~m}^{2}$ of the irregular water atomized powder and the $0.023 \times 10^{-12} \mathrm{~m}^{2}$ of the dendritic electrolytic powder, and the corresponding ratio was $99.2: 12.1: 1$. Since the effect of density variation had been eliminated, the next main possible variation causing the great difference in permeability was the particle size. However, the particle sizes of the gas atomized, water atomized, and electrolytic powders were 65.4, 46.2, and $21.4 \mu \mathrm{m}$, respectively, and the ratio was $3.1: 2.2: 1$. Using the square of this particle size ratio and eq. (1), the large difference in the permeability, as shown in Fig. 4, or the ratio of $99.2: 12.1: 1$, could not be explained satisfactorily. The pore sizes, which are related to the particle sizes, of these three sintered compacts were then examined using a mercury porosimeter. The average pore diameters were $17.16,8.18$, and $3.27 \mu \mathrm{m}$ for the spherical, irregular, and dendritic powders, respectively, as shown in Fig. 5. The 
(a)

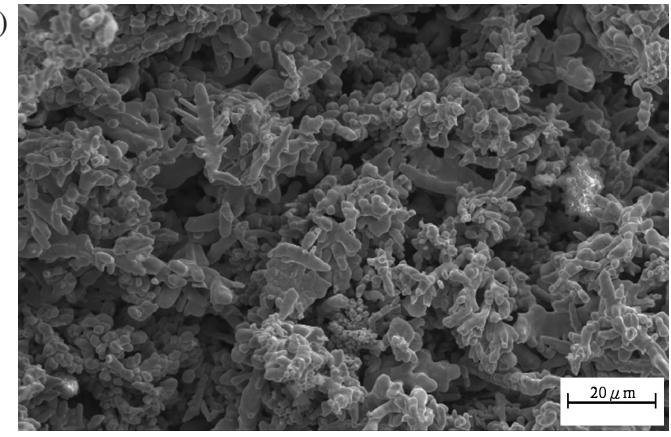

(b)

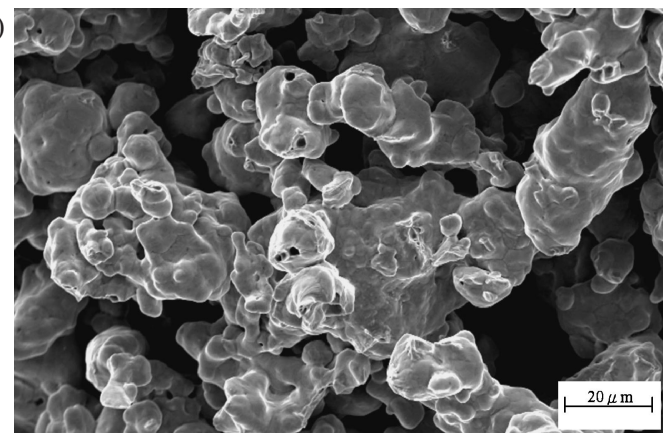

(c)

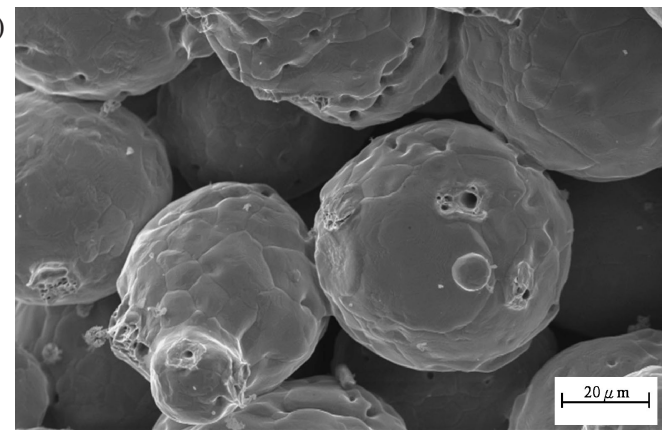

Fig. 6 The morphology of the pore structure in the compacts sintered at 1073 K. (a) Dendritic, (b) irregular, and (c) spherical powder.

ratios of these pore sizes were still too small to explain the large differences in the measured permeabilities.

Since the porosity and the particle size, which is related to the pore size, have been considered in eq. (1), the large difference in permeabilities must be attributed to the geometrical factor of the pore structure. Since water atomized powder and electrolytic powder could produce more irregular pore shapes and rougher pore surfaces, as shown in Fig. 6, it is not surprising that their geometrical factors, $C$, are very different.

\subsection{Effect of powder shape on capillary speed}

The capillary speed has been recommended for evaluating whether a copper powder is suitable for heat pipe applications because it is directly related to the water supply rate. ${ }^{6)}$ To measure the capillary speed, the loosely packed powders in the stamped container were sintered at $1123 \mathrm{~K}$ for $3.6 \mathrm{ks}$ in hydrogen, yielding sintered densities of $2.29,4.18$, and $5.48 \times 10^{3} \mathrm{~kg} / \mathrm{m}^{3}$ for dendritic, irregular, and spherical powders, respectively. Using the capillary rise heights of these flat specimens, Fig. 7 shows that the spherical powder yielded the fastest climb rate, followed closely by the

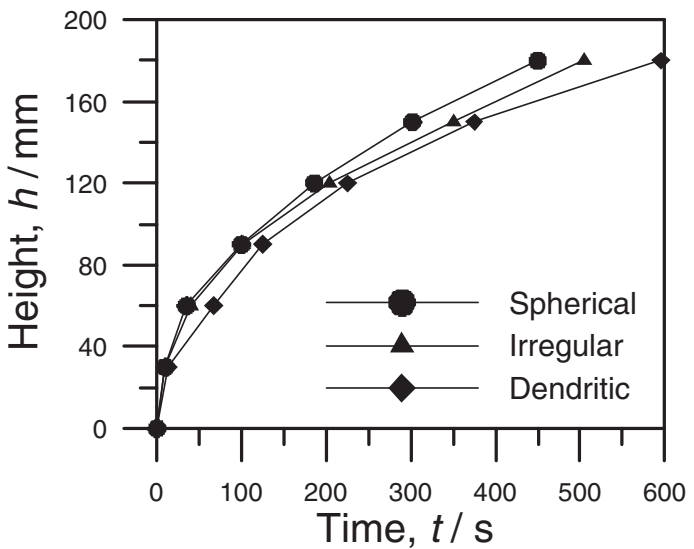

Fig. 7 The capillary rise heights of the flat specimens that were prepared using powders with different shapes.

irregular powder and the dendritic powder. The slopes of these curves are the capillary speeds and are shown in Fig. 8. Also plotted are the theoretical curves calculated using eq. (10) with a tortuosity factor $(\tau)$ of $\sqrt{2}$ and a particle shape factor $(\lambda)$ of 1 and 2 . The experimental data show that the capillary speed of the spherical powder can be represented by the equation with $\lambda=1$. For irregular and dendritic powders, which had large geometrical factors ( $C$ in eq. (1)) due to the more irregular pore structures and the rougher pore surfaces, as shown in Fig. 6, the measured capillary speed data agree with the curves calculated using a higher particle shape factor $(\lambda)$. For the irregular powder, the measured data were close to the curve with $1<\lambda<2$. For dendritic powder, the data were close to the curve with $\lambda=2$, as shown in Fig. 8. These good agreements suggest that eq. (10) can be used to estimate the capillary speed of water in sintered porous wicks, not only for spherical powders but also for nonspherical powders.

It is also noted in Fig. 8 that the differences in the capillary speed of the three powders were not significant, within a factor of 1.33 , despite their large differences in permeability, with a ratio of $2.3: 1: 13.2(0.80,0.34$, and $4.50 \times 10^{-12} \mathrm{~m}^{2}$ ) for the dendritic, irregular and spherical powders, respectively, as were measured on the cylindrical compacts that were sintered at $1123 \mathrm{~K}$. To check if this discrepancy in the correlation between capillary speed and permeability was related to the capillary pressure, the mean pore radii of the cylindrical compacts prepared using the dendritic, irregular, and spherical powders were measured using a mercury porosimeter and determined to be 8.9, 9.2, and $22.1 \mu \mathrm{m}$, respectively, as shown in Fig. 9. The small pore radii of the dendritic and irregular powder compacts, which give high capillary pressure, must have counteracted their low permeability and thus only show a small difference in capillary speed as compared to that of the spherical powder. This indicates that the heat dissipation of heat pipes cannot be determined solely by the permeability or capillary pressure. The capillary speed, which takes into account both permeability and capillary pressure and is directly related to the water supply rate, is considered a better method for the evaluation of copper powders for heat pipe applications. 
(a) ${ }^{\top}$

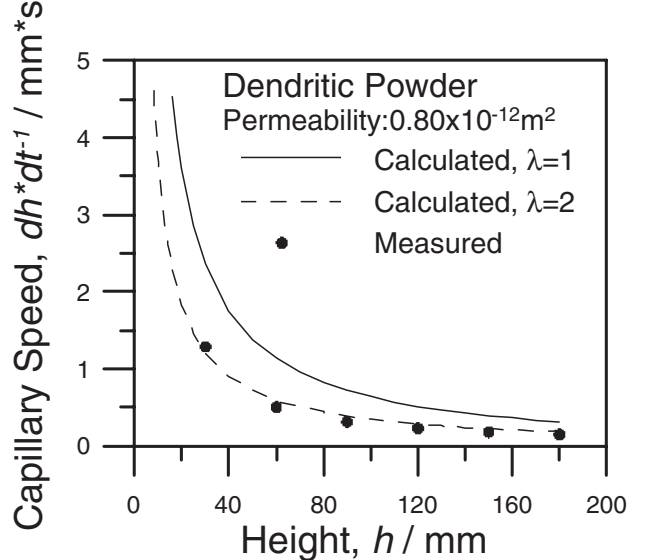

(b) $\bar{c}$

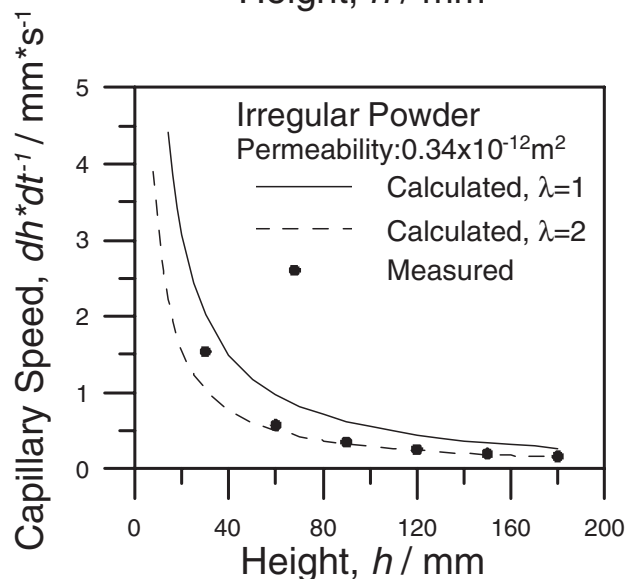

(c) $\bar{T}$

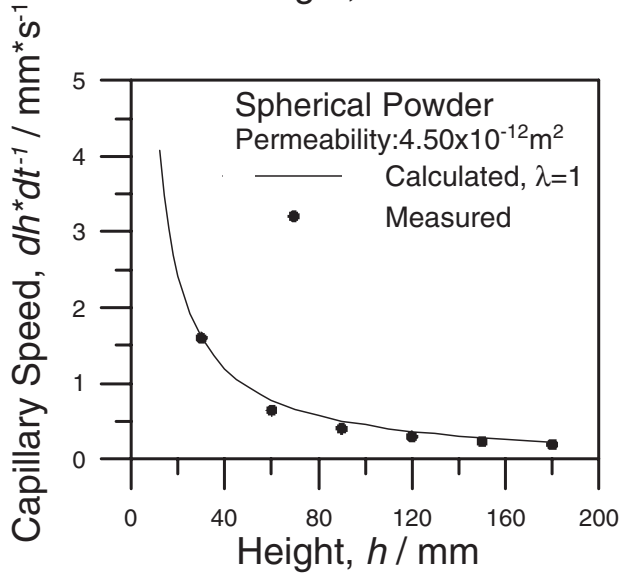

Fig. 8 The capillary speeds of the flat specimens that were prepared using powders with different shapes. (a) Electrolytic powder with dendritic shape, (b) water atomized powder with irregular shape, and (c) gas atomized powder with spherical shape.

\subsection{Effect of processing parameters on permeability}

Figure 10 shows that the permeability of the high temperature sintered (HT) sample, $3.31 \times 10^{-13} \mathrm{~m}^{2}$, was higher than that of the compacted and low-temperaturesintered (LT) sample, $1.10 \times 10^{-13} \mathrm{~m}^{2}$. Since both HT and LT specimens used the same water atomized powder and their sintered densities were the same, $4.80 \times 10^{3} \mathrm{~kg} / \mathrm{m}^{3}$, the difference in the measured permeability must be caused by the different $C$ values in eq. (1), which could be influenced by factors such as surface roughness and effective pore length. The calculated $C$ 's of the HT and LT specimens were 2249 and 6768, respectively. These differences can be

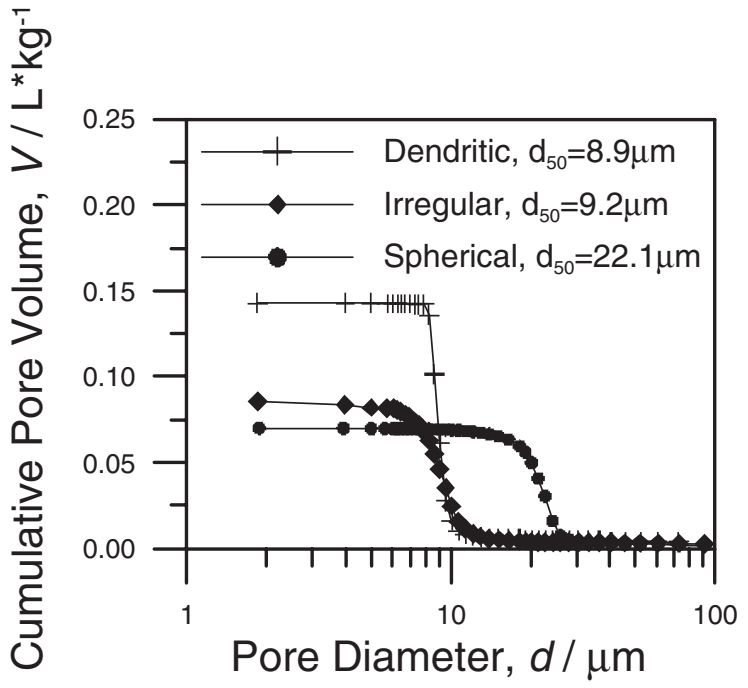

Fig. 9 The pore size distribution of the loose-powder-sintered wicks in the cylindrical compact, showing that spherical powders produce the largest pore size.

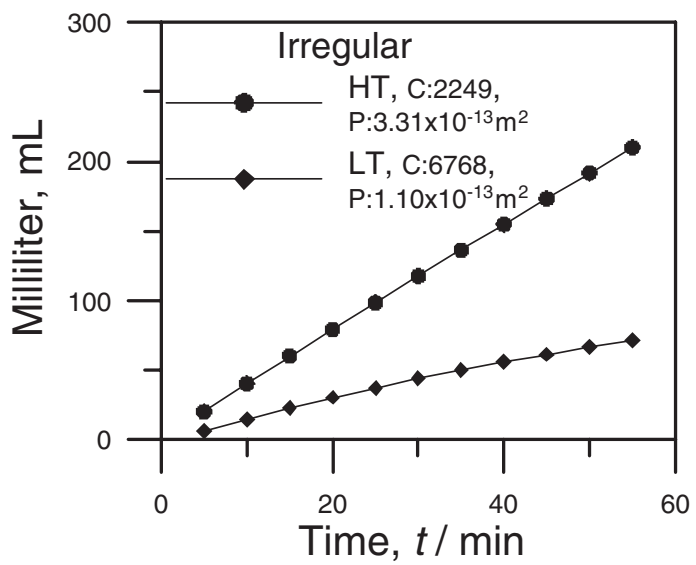

Fig. 10 The flow rate of water in the sintered LT and HT wicks.

rationalized in Fig. 11, in which the LT specimen shows a much rougher powder surface than that of the HT specimen. These morphology differences caused the difference in the effective pore size, as measured using the mercury porosimeter. As illustrated in Fig. 12, the LT specimen had a smaller pore size of $4.80 \mu \mathrm{m}$, while the HT specimens had a larger pore size of $6.43 \mu \mathrm{m}$ and a slightly narrower pore size distribution, both of which are favorable for high permeability. The bubble test also showed that the HT sample had a maximum pore size of $10.28 \mu \mathrm{m}$, larger than the $7.65 \mu \mathrm{m}$ of the LT sample, both of which are slightly higher than those of the porosimetry data.

Since both the HT and LT specimens were prepared with the same water atomized powder and the sintered densities were the same, their pore shape, pore length, and tortuosity should not be very different. The above results of permeability and geometrical factor, $C$, of the HT and LT specimens along with the data in section 3.1 therefore suggest that the surface roughness could be the most important factor in determining the geometrical factor of sintered porous material. 
(a)

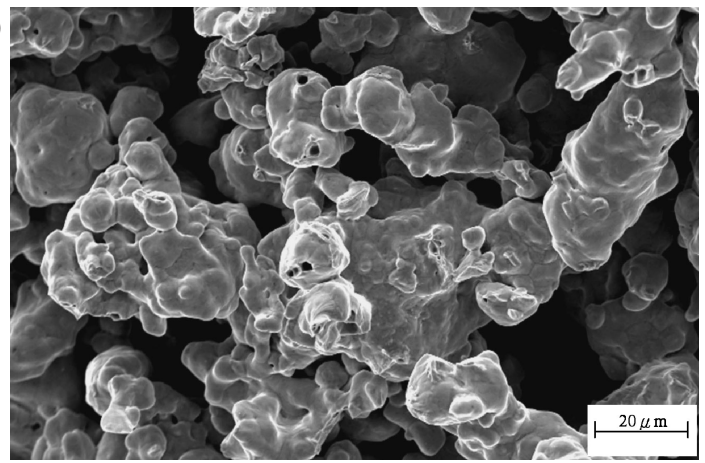

(b)

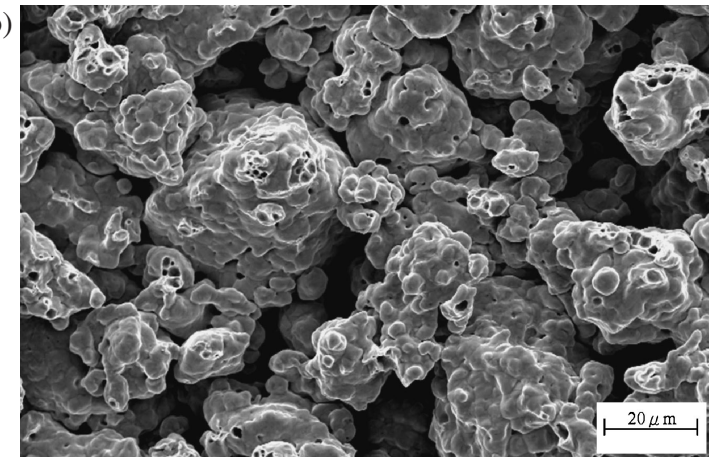

Fig. 11 The morphology of (a) HT and (b) LT sintered compacts, showing the difference in the surface roughness.

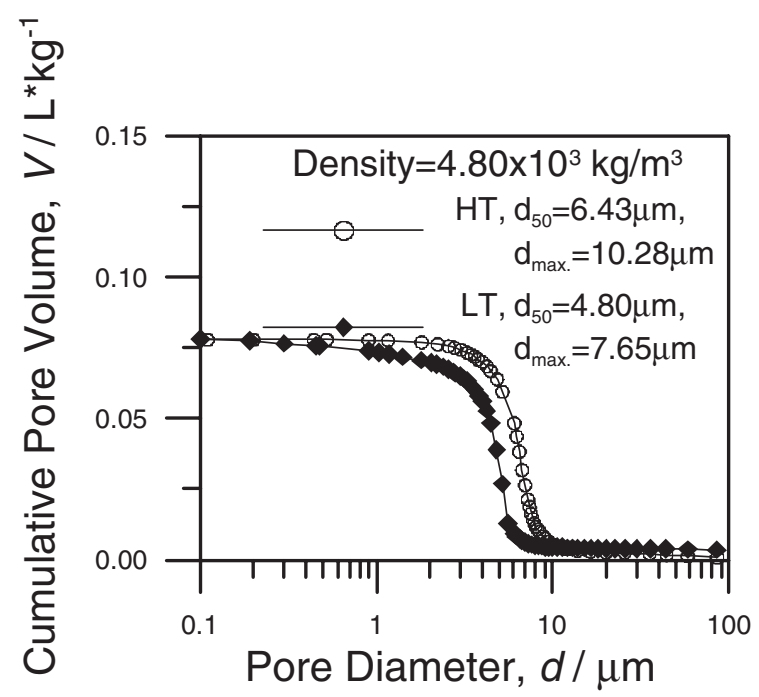

Fig. 12 The pore size distribution of the loose-powder-sintered wick (HT) and compacted and sintered wick (LT).

\subsection{Effect of powder shape on heat dissipation}

The flat specimens that were used for capillary speed measurement were also used for the heat dissipation measurement. Under the fixed power input of 50 watts, the specimen that was made with the spherical powder had the lowest temperature at the evaporator zone, and the specimen made with the dendritic powder had the highest, as shown in Fig. 13. The effect of powder shape on heat dissipation was also demonstrated by measuring the water heights of the flat specimens to see if the dry-out phenomenon occurred. Figure 14 shows that dry-out occurred on all powders, with the dendritic powder the most

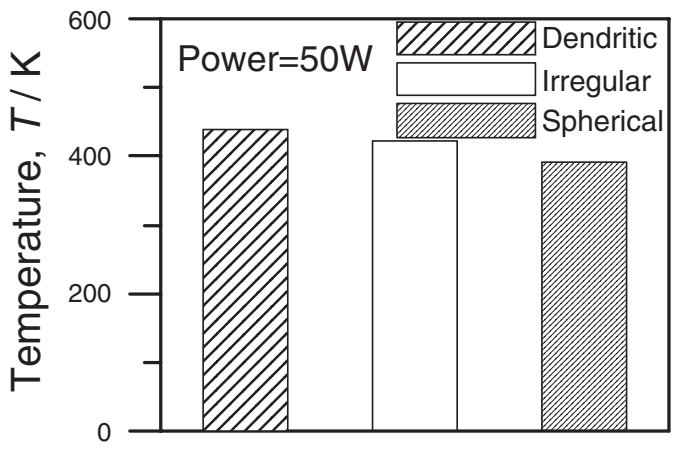

Fig. 13 The temperatures at the evaporator zone of the sintered wicks that were prepared using powders with different shapes.

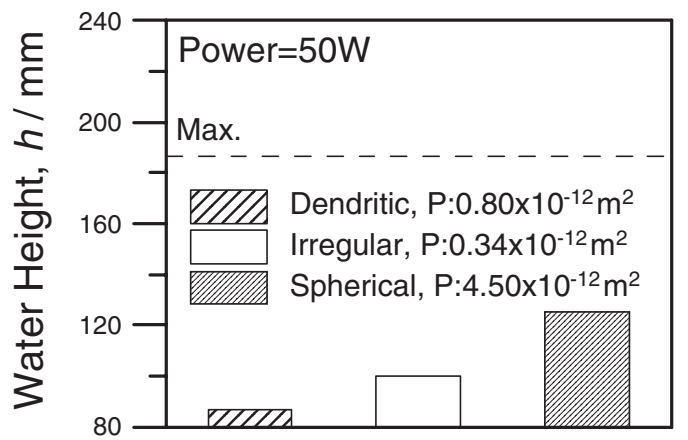

Fig. 14 The water heights of the specimens that were prepared using powders with different powder shapes.

serious, followed by the irregular and spherical powders. However, the difference was small, similar to that observed in the capillary speed.

Calculations using eq. (2) indicated that the capillary pressure is usually high enough to draw water from the condenser section to the evaporator section in most heat pipes. Thus, permeability has become the main factor in determining the heat dissipation performance in many cases. ${ }^{1-4,6)}$ But the heat dissipation results of this study show that the dendritic powder has a permeability of $0.80 \times 10^{-12} \mathrm{~m}^{2}$, higher than the $0.34 \times 10^{-12} \mathrm{~m}^{2}$ of the irregular powder, as shown in Table 3 , but a poorer thermal performance. This shows that permeability alone cannot be used to predict the heat dissipation of a heat pipe. This study demonstrates that capillary speed is a better method for determining the suitability of a copper powder for heat pipe applications.

\section{Conclusions}

The effects of powder shape, compacting pressure, and sintering temperature on the permeability, capillary speed, and thermal performance of sintered heat pipes were examined. The following are the concluding remarks.

(1) With the loose powder sintering process, the gas atomized powder with a spherical shape showed the highest permeability, capillary speed, and the best heat dissipation, followed by water atomized powder with an irregular shape and electrolytic powder with a dendritic shape. 
Table 3 Comparison of the tap density, sintered density, permeability, and capillary speed of the loose-powder-sintered flat specimen.

\begin{tabular}{lccccc}
\hline & $\begin{array}{c}\text { Tap Density, } \\
\mathrm{kg} / \mathrm{m}^{3}\end{array}$ & $\begin{array}{c}\text { Sintered Density, } \\
\mathrm{kg} / \mathrm{m}^{3}\end{array}$ & $\begin{array}{c}\text { Porosity, } \\
\%\end{array}$ & $\begin{array}{c}\text { Pore Size*, } \\
\mu \mathrm{m}\end{array}$ & \multicolumn{2}{c}{$\begin{array}{c}\text { Capillary Speed } \\
\mathrm{mm} / \mathrm{s}\end{array}$} \\
\hline Dendritic & $2.17 \times 10^{3}$ & $2.29 \times 10^{3}$ & 74.4 & 8.94 & 0.30 \\
Irregular & $3.52 \times 10^{3}$ & $4.18 \times 10^{3}$ & 53.3 & 9.19 & 0.80 \\
Spherical & $5.62 \times 10^{3}$ & $5.48 \times 10^{3}$ & 38.8 & 22.09 & 0.36 \\
\hline
\end{tabular}

*measured using the loose-powder-sintered cylindrical compact

** measured at the top position of the vertical flat specimen

(2) The capillary speed was shown to have a good correlation with the heat dissipation results and is favorable over the permeability or capillary pressure for evaluating whether a copper powder is suitable for heat pipe applications or not.

(3) The capillary speeds of the three powders are quite close, within a factor of 1.33 , in spite of the big difference, up to 13.2 times, in their permeability. This is because the dendritic and irregular powders provide high capillary pressures that offset their low permeabilities and thus have capillary speeds close to that of the spherical powder.

(4) The geometrical factors in the Kozney-Carman equation were 250, 1445, and 3108, for compacts with similar permeability that were made from spherical, irregular, and dendritic powder, respectively. The large deviations from the geometrical factor of 180 of the perfectly spherical powder indicate that the effect of powder shape on the permeability could be even more important than that of the particle size and porosity in the equation.

(5) The permeability could be improved by providing a smooth pore surface, as was demonstrated by using the same water atomized powder with different compacting pressures and sintering temperatures. This suggests that the surface roughness could be more important than the pore length, tortuosity, and pore shape in determining the geometrical factor, $C$, in the Kozney-Carman equation.

\section{REFERENCES}

1) A. Faghri: Heat Pipe Science and Technology, (Taylor and Francis, Washington, DC, 1995) pp. 1-131.

2) B. Holley and A. Faghri: Appl. Therm. Eng. 26 (2006) 448-462.

3) D. A. Pruzan, L. K. Klingensmith, K. E. Torrance and C. T. Avedisian: Int. J. Heat Mass Transfer 34 (1991) 1417-1427.

4) K. C. Leong, C. Y. Liu and G. Q. Lu: J. Porous Mater. 4 (1997) 303-308.

5) A. Lorenz, E. Sachs and S. Allen: Metall. Mater. Trans. A 35A (2004) 641-653.

6) Y. J. Lin and K. S. Hwang: Metall. Mater. Trans. A 40 (2009) 20712078.

7) E. O. Einset: J. Am. Ceram. Soc. 79 (1996) 333-338.

8) D. Muscat and R. A. L. Drew: Metall. Mater. Trans. A 25A (1994) 2357-2370.

9) K. A. Semlak and F. N. Rhines: Trans. Metall. Soc. AIME 212 (1958) 325-31.

10) K. P. Trumble: Acta Mater. 46 (1998) 2362-2367.

11) J. Bear: Dynamics of Fluids in Porous Media, (Dover Publications, Inc., New York, NY, 1988) pp. 161-67.

12) R. M. German: Powder Injection Molding, (MPIF, Princeton, NJ, 1990) pp. 286-291.

13) C. N. Satterfield: Heterogeneous Catalysis in Practice, (McGraw-Hill Book Co., New York, NY, 1980) p. 336.

14) T.-S. Sheu, P.-P. Ding, I.-M. Lo and P.-H. Chen: Exp. Therm. Fluid Sci. 22 (2000) 103-110. 